\title{
Demonstration of a Multipulse Interferometer for Quantum Kicked-Rotor Studies
}

\section{Citation}

Tonyushkin, Alexey A., Saijun Wu, and Mara Prentiss. 2009. Demonstration of a multipulse interferometer for quantum kicked-rotor studies. Physical Review A 79(051402).

\section{Published Version}

doi:10.1103/PhysRevA.79.051402

\section{Permanent link}

http://nrs.harvard.edu/urn-3:HUL.InstRepos:10591635

\section{Terms of Use}

This article was downloaded from Harvard University's DASH repository, and is made available under the terms and conditions applicable to Open Access Policy Articles, as set forth at http:// nrs.harvard.edu/urn-3:HUL.InstRepos:dash.current.terms-of-use\#OAP

\section{Share Your Story}

The Harvard community has made this article openly available.

Please share how this access benefits you. Submit a story.

Accessibility 


\title{
Demonstration of a multipulse interferometer for quantum kicked-rotor studies
}

\author{
A. Tonyushkin, ${ }^{*} \mathrm{~S} . \mathrm{Wu},{ }^{\dagger}$ and M. Prentiss \\ Department of Physics, Harvard University, Cambridge, Massachusetts 02138, USA
}

(Received 11 June 2008; published 27 May 2009)

\begin{abstract}
We implemented a multipulse interferometer scheme that allows us to study a quantum kicked rotor by observing dephasing of momentum coherence. Our study shows that momentum coherence can be nearly perfectly preserved under conditions where the mean energy as a function of the kick number is known to increase without bound. The accompanying width narrowing of these coherences may provide a new method for accurate measurement of the recoil frequency.
\end{abstract}

DOI: 10.1103/PhysRevA.79.051402

PACS number(s): 32.80.Qk, 03.75.Dg, 05.45.Gg, 37.25.+k

Coherence and interference are fundamental properties of quantum mechanical systems that cause quantum dynamics to be different from classical dynamics. With the advent of the atomic delta kicked rotor (ADKR) [1] it has been possible to probe a boundary between classical and quantum dynamics in a single system. These studies showed predicted phenomena such as quantum resonances $[1,2]$ as well as unexpected ones such as quantum accelerator modes $[3,4]$, dynamical localization of momentum $[5,6]$, and chaos-assisted tunneling [7]. The stability [8] and scaling [9] of ADKR features are still an open field for both theoretical and experimental studies that not only promise to answer fundamental questions about quantum mechanics but could offer novel schemes for atom-optics-based sensors.

The conventional ADKR implementation consists of a system of cold atoms periodically perturbed by pulsed offresonant standing waves; the system dynamics is studied by observing the momentum distribution and the mean energy. In previous interferometry approaches the ADKR was applied to momentum states, but the interferometer output probed the coherence between internal states that were not directly affected by the ADKR [4]. In contrast, we offer a scheme for experimental study of the effect of a kicked rotor on atomic coherences by measuring the dephasing induced by an ADKR that acts on the momentum states whose coherence is measured by the output of an external state atom interferometer [10]. With our scheme we are able to experimentally realize the main and fractional quantum resonances for momentum coherences and study them in the broad range of perturbation with high resolution. Our results provide a surprisingly close match to the corresponding features in the mean energy of previous ADKR realizations. We demonstrate that the coherence between different momentum states that is established by the interaction with the first optical grating in the atom interferometer can be nearly perfectly preserved at a quantum resonance (QR) while additional fringes exist in the vicinity of QR [11]. We directly show that fractional QR corresponds to higher order momentum coherences in the atom interferometer. Our method is also suitable to investigate the scaling of recently predicted fractional resonances of a $\delta$-kicked accelerator for thermal atoms [12],

\footnotetext{
*alexei@nyu.edu

†Present address: NIST, Gaithersburg, MD 20899.
}

which is attractive for accurate measurement of gravitational acceleration due to the lack of mean-field effects. Finally, we show that our system may be used as a tool for precision measurements of atomic recoil frequency [13].

The experimental setup and matter-wave diffraction scheme are shown in Fig. 1. In our experiment we subject a cloud of cold ${ }^{87} \mathrm{Rb}$ atoms in a magnetic guide to periodic kicks from a sinusoidal potential created by standing wave (SW) pulses of off-resonant laser light along the guiding direction. A detailed description of the de Broglie wave interferometer in a magnetic guide is given elsewhere [14]. The interferometer SW consists of the two counterpropagating traveling waves with $k$ vectors $\mathbf{k}$ precisely aligned along the guide and the SW field is $\sim 20 \Gamma$ blue detuned from $F=1$ $\rightarrow F^{\prime}=2$ transition. The schematic of matter-wave diffraction in the atom interferometer is conveniently described by the recoil diagram (see, for example, Refs. $[15,16]$ ) in Fig. 1(b). We realize the ADKR potential by applying a train of offresonant $\mathrm{SW}$ pulses in between the two interferometer pulses $\left[\mathrm{SW}_{1}\right.$ and $\mathrm{SW}_{2}$ in Fig. 1(b)]; therefore we treat a $\delta$-kicked rotor as a perturbation to the matter wave's dynamics in a de Broglie wave interferometer. The long trapping time and corresponding long coherence time $(\sim 60 \mathrm{~ms})$ of the atom interferometer in a magnetic guide [14] allow us to investigate ADKR for the large number of pulses $N$ and over a broad range of kicking strengths, characterized by the pulse area $\theta$. For sufficiently short kicks, the motion of atoms during pulse

(a)

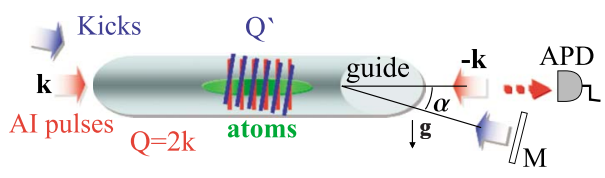

(b)

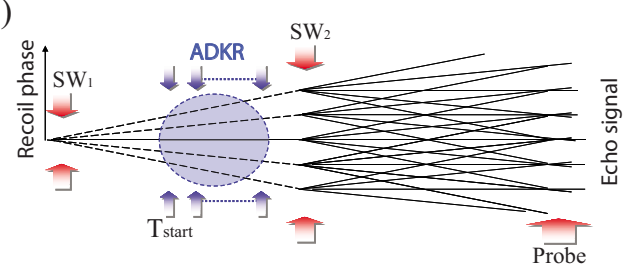

FIG. 1. (Color online) Schematic diagram of experimental setup: (a) configuration along the magnetic guide; (b) matter-wave recoil diagram. Here, $\mathrm{AI}=$ atom interferometer, $\mathrm{APD}=$ avalanched photodiode, $\mathrm{ADKR}=$ atomic $\delta$-kicked rotor, and $\mathrm{SW}_{1,2}=$ first and second standing waves of AI. 


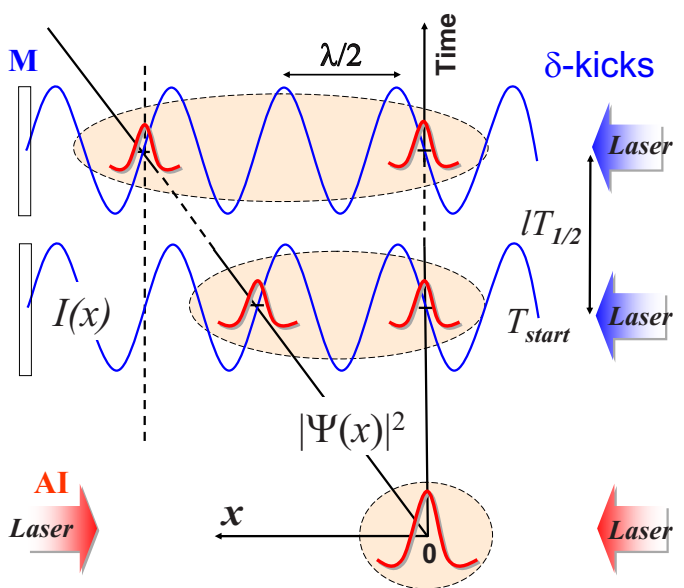

FIG. 2. (Color online) A qualitative diagram of wave-packet dynamics with coherence preservation. At time $t=0$ the wave packet (shown in red) is diffracted by the interferometer SW pulse into many (only two shown for clarity) wave packets which at time $T_{\text {start }}$ interact with kicking SW (shown by blue arrows). Here, $I(x)$ laser intensity pattern created by retroreflection from the mirror M.

duration $t_{p} \approx 0.5 \mu$ s can be neglected. Also for a pulse duration much less than the kick period $T_{0}$ one can approximate each kick as a delta function [6]. The kicking pulses are 6.8 $\mathrm{GHz}$ red detuned from the upper manifold of ${ }^{87} \mathrm{Rb} D_{2}$ and the maximum achievable pulse area $\theta \approx 9.5$. As shown in Fig. 1(a), the propagation direction of the kicked-rotor SW is tilted by up to $40 \mathrm{mrad}$ angle with respect to the interferometer SW. The grating vectors $Q$ and $Q^{\prime}$ of the respective interferometer and kicked-rotor SW fields are not equal. Our detection method relies on coherent backscattering of the probe pulse from the atomic grating induced by the interferometer pulses; therefore, it is only sensitive to spatial distributions with the periodicity $2 \pi / Q$ of the interferometer SW. Thus, in order to be detected the final coherence has to be converted to $2 \pi / Q$.

The ADKR Hamiltonian is presented elsewhere [1]. A schematic diagram of the interaction of a kicked rotor with matter waves is given in Fig. 2. The atomic wave packet is split by the interferometer SW pulse at time $t=0$ into wave packets with momenta that differ by integer multiples of $\hbar Q$; thus the momentum coherence is created. The interaction with the kicked-rotor potential begins at a time $t=T_{\text {start }}$ when the first kicking SW with a grating vector $Q^{\prime}$ is applied. If the spatial displacement between any two diffracted wave packets at the time of interaction is commensurate with the spatial period of the kicking SW pulse then those wave packets experience the same potential; therefore, the differential phase between the wave packets is equal to zero and the interferometer signal is unaffected by this interaction [17]. The same argument applies for interactions at times $\left\{T_{\text {start }}\right.$ $\left.+l T_{1 / 2}\right\}$, where $l$ is an integer, $T_{1 / 2}=\pi / \omega_{Q^{\prime}}$ is the half-Talbot time $\left(T_{1 / 2} \approx 33.2 \mu \mathrm{s}\right.$ for $\left.{ }^{87} \mathrm{Rb}\right)$, and $\omega_{Q^{\prime}}=\hbar Q^{\prime 2} / 2 m$ is a twophoton atomic recoil frequency for the kicked-rotor potential. As compared with the resonances at the other $T_{\text {start }}$ that require regular spacing and strength of the applied SW pulses [18], in our case the invariance under the ADKR perturbation does not rely on the temporal pattern of the kicks (a)

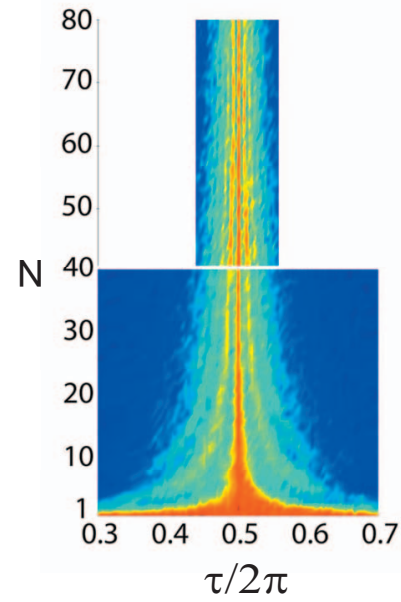

(b)

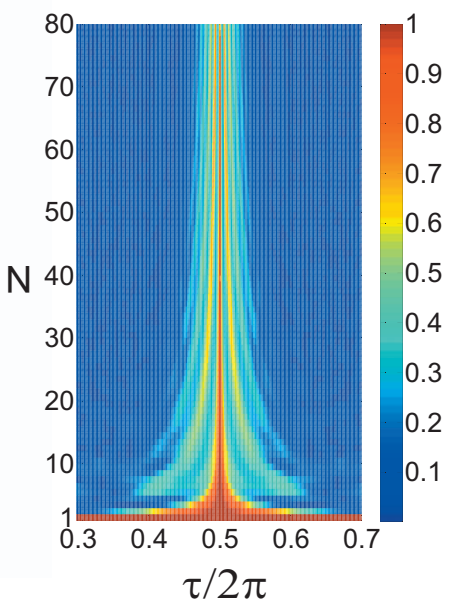

FIG. 3. (Color) Matter-wave coherence dynamics: (a) experimental data and (b) corresponding simulations $(\theta=0.7)$. The data show the coherence preservation (red color on the contour plot) in the narrow vicinity of the kicking period $\tau=\pi\left(T_{0} \approx 33.2 \mu \mathrm{s}\right)$ : the horizontal axis is the normalized kicking period and the vertical axis is the number of kicks.

and is still valid for the infinitely large single kick or random strength kicks separated by irregular integer multiples of $T_{1 / 2}$. To distinguish between other types of quantum resonances we call the latter a robust resonance in a similar fashion as was done for mean energy QR [19]. To study the dephasing of the interferometer signal due to ADKR we use a normalized echo amplitude $A$, given by the first Fourier harmonic of the atomic density $\rho_{-Q}(T)$ which is obtained by the backscattering technique. The normalized echo amplitude $A=\rho_{-Q}^{\Delta}(T) / \rho_{-Q}(T)$ depends on the parameters of perturbation potential $\Delta$ and it is independent of the parameters of atom interferometer. For the kicked SW with the period $l T_{1 / 2}$ the wave-packet spatial displacement is given by $X\left(T_{\text {start }}\right)$ $=\delta x\left(T_{\text {start }}\right)+2 l \pi / Q^{\prime}$, where $\delta x\left(T_{\text {start }}\right)$ is the initial spatial displacement. The robust resonance condition introduced above (also see Fig. 2) corresponds to $\delta x\left(T_{\text {start }}\right)$ which is commensurate to the period $2 \pi / Q^{\prime}$ of the kicking SW. Therefore, we can write the displacement as $X\left(T_{\text {start }}^{\mathrm{res}}\right)=2(l+m) \pi / Q^{\prime}$ for $l, m$ integers. The differential strength defined as [18] $\phi$ $=2 \theta \sin Q^{\prime} X\left(T_{\text {start }}\right) / 2$ is equal to zero for such a condition, therefore providing the invariance of the coherence signal on the perturbation strength $\theta$ at any given kick.

In each experiment we fix the total interferometer time $T \leq 20 \mathrm{~ms}$ that corresponds to the optimal "echo" signal and record its amplitude; then we turn on the perturbation potential and record the signal as we modify one or several parameters of perturbation. Figure 3 shows the effect of the ADKR on the coherence between the momentum states that differ by $2 \hbar k$ for up to $N=80$ kicks applied and the pulse area of the kicking SW $\theta=0.7$, as determined by fitting the data to simulation curve with no free parameter. The experimental data are given in Fig. 3(a) where for each fixed $N$ we measured the backscattered echo signal at time $T$ as a function of the normalized kicking period $\tau=\omega_{Q^{\prime}} T_{0}$. In practice $\omega_{Q}-\omega_{Q^{\prime}} \ll \omega_{Q}$, so for convenience we ignore the difference and use $T_{1 / 2}=\pi /\left\langle\omega_{Q^{\prime}}\right\rangle=33.2 \mu$ s. The experimental scan as a 
(a)

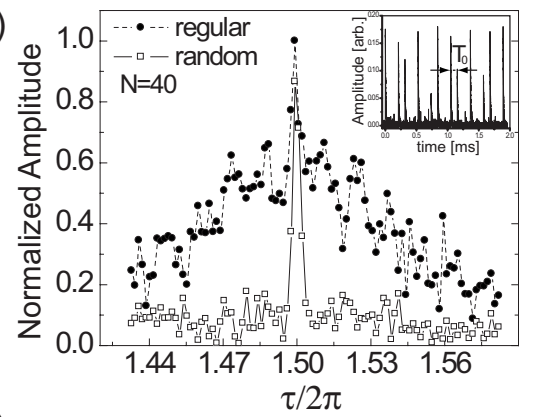

(b)

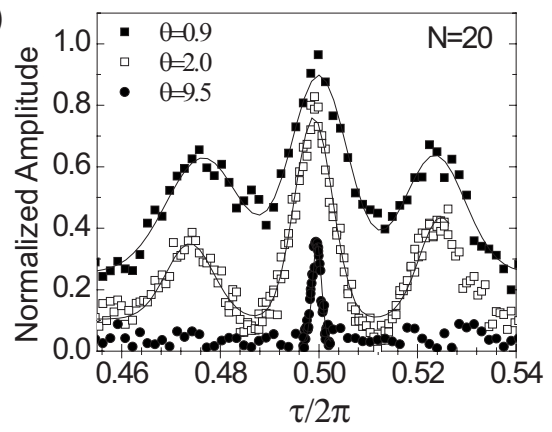

FIG. 4. Robustness of coherence resonances: (a) coherence amplitude at $N=40$ vs kicking period corresponding to the regular (circles) and random (open squares) kicking sequences (the curves are used to guide the eyes); the inset shows an example of random amplitude kicking pattern as monitored by fast photodiode; (b) resonance peak with fringes at finer time scale $\left(T_{0}\right.$ $=33.2 \pm 2.7 \mu \mathrm{s})$ for regular kicks at $N=20$ and $\theta=0.9,2.0,9.5$, where high pulse area produces fringes with higher contrast (the solid curves correspond to multiple peak fits).

function of the kicking period consists of two parts: coarse for $N=1-40$ and fine for $N=41-80$, where for greater detail we scanned the kick period with higher resolution in the narrow vicinity of the resonance $\tau / 2 \pi \in[0.44,0.55]$. The corresponding results of numerical simulations are shown in Fig. 3(b). The normalized signal $A$ is represented by false color scaled according to the color bar where red corresponds to unity. It is seen from the data that the coherence gets depleted everywhere except a narrow vicinity of the resonance where it remains unity. The delay time $T_{\text {start }}$ is chosen so that the separation between the two atomic wave packets is an integer multiple of $\pi / Q^{\prime}$. We precisely determine the required $T_{\text {start }}$ which corresponds to a maximum of an echo amplitude from the recoil oscillations experiment where $A$ is plotted versus $T_{\text {start }}$. In agreement with the qualitative picture given in Fig. 2, both simulations and experiment show that the lowest order coherence is nearly perfectly preserved for the main QR condition $\tau=\pi$. In fact, we experimentally confirmed this result for even and odd integer multiples of the main resonance up to $\tau=37 \pi$. Our results agree with the recently observed similar behavior for mean energy resonances for up to $20 \pi$ [20].

In addition to the central peak at the resonance the data (Fig. 3) show finer fringes. For sufficient power the contrast of these fringes is close to $100 \%$. Similar oscillations around the main resonance were predicted [19] and observed [11] for the mean energy. In our case we relate these fringes to a complicated interference of matter waves on the $N$ scattering (a)

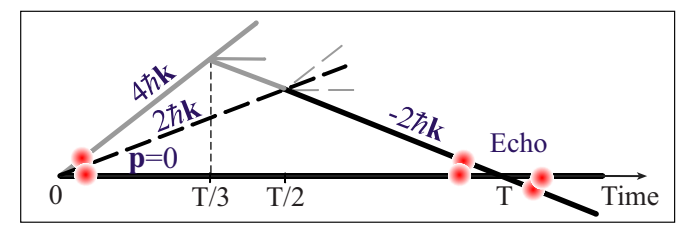

(b)

(c)
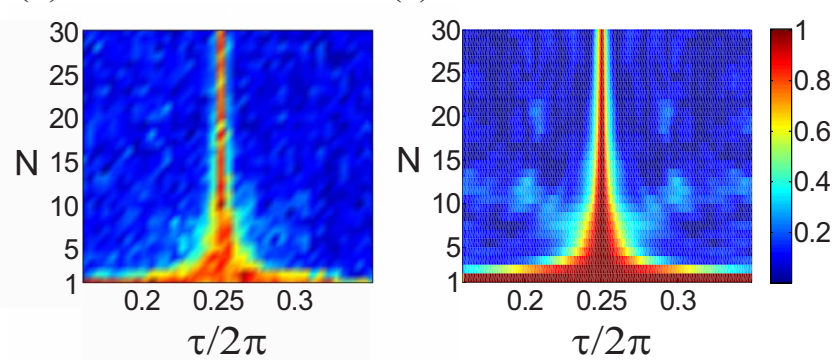

FIG. 5. (Color online) Effect of ADKR on higher order coherence (fractional quantum resonance): (a) the recoil diagram for one representative loop of the second echo scheme as compared to the first echo scheme (dashed line); (b) experimental data; and (c) simulations $(\theta=1)$. On the contour plots the color represents the amplitude of interference signal, the horizontal axis is the normalized kicking period, and the vertical axis is the number of kicks.

nodes. The observed signal as a function of time is analogous to the intensity pattern as a function of angle for light diffracted by a double-slit grating, where the slit separation is proportional to $N$ and relative transmission of two slits is weighed by $\theta$. In agreement with the above support model, we found both experimentally and from numerical simulations that the fringe period and the width of a fringe scale as $\sim 1 / N$. If the kicking strength is sufficiently small, these fringes wash out producing high background around the sharp robust resonance peak. Even for $\tau \sim \pi$ the background can be removed by either applying high pulse area optical pulses or by randomizing the strengths of successive low power pulses [19]. Figure 4(a) shows the removal of the background due to application of random amplitude kicks generated by an optical chopper, which randomly blocks the kicked-rotor light. For the presented data the kicking period was scanned near the third resonance $\tau=3 \pi$ and the chopping period was of the same order but incommensurate with it. Though the background is removed, the coherence of the robust central resonance is preserved. The removal of the background by high strength regular kicks is illustrated in Fig. 4(b), where the number of identical kicks is fixed at $N$ $=20$. At the highest $\theta \approx 9.5$, the coherence loss due to spontaneous emission becomes important, resulting in a decrease in the robust resonance height.

In general, only the coherences due to momentum difference $2 \hbar k$ are considered in the so-called "first echo" scheme, as shown in Fig. 1(b), where interferometer pulses are applied at times $t=0$ and $T / 2$. Atomic fringe patterns due to the second-order coherences were observed using similar detection method in Ref. [16] and using an optical mask technique in Ref. [21]. In our experiment we apply ADKR when the different momentum states are separated in phase space by different amounts, so the detected signal encodes the information of the effect of ADKR on higher order coherences. 
This effect results in the formation of fractional quantum resonances which were recently observed with Bose-Einstein Condensate and ultracold atoms in an optical lattice with velocity selection [22]. To realize such fractional resonance we employed a so-called "second echo" configuration [15] as shown in Fig. 5(a) where the two interferometer SW pulses are applied at times $t=0$ and $T / 3$ and the lowest order coherences correspond instead to a $4 \hbar k$ momentum difference. Figures 5(b) and 5(c) show experimental and simulation data, respectively, of the second-order coherence evolution for up to $30 \mathrm{kicks}$ near $\tau / 2 \pi=0.25\left(T_{0} \approx 16.6 \mu \mathrm{s}\right)$. We note that there is no quantum resonance at $\tau / 2 \pi=0.25$ for the first echo scheme. An arbitrary high order fractional QR at integer multiples of $\tau=\pi / n$ can be probed similarly in the $n$ th-echo configuration with the second interferometer pulse applied at $t=T /(n+1)$ for integer $n$.

The described robust resonance phenomena could be used for precision measurements of atomic recoil frequency $\omega_{Q^{\prime}}$ and hence $\hbar / m_{\mathrm{Rb}}$ [13]. The sharpness of the resonance peak (see Fig. 4) can be used to determine precisely the resonance period and thus the recoil frequency. The precision of such a measurement is defined by $\Delta \omega_{Q^{\prime}} / \omega_{Q^{\prime}}=\Delta T_{1 / 2} /\left(l T_{1 / 2}\right)$ for an integer $l$. Theoretical analysis of the resonance width shows that it should scale as $\Delta T_{1 / 2} \sim \delta T_{0} /\left(l \theta N^{p}\right)$, where $\delta T_{0}$ is the width after single kick and $p$ is a decay factor. For high enough $N$ we obtained $p=1$ [23]. With experimentally feasible parameters the estimated precision is better than several parts per million for single run, which, at this level, is not affected by systematic effects described in Ref. [24]. In order to reach high precision, however, it is necessary to use the same SW for interferometer and kicking pulses; there is also a stringent requirement on the optical beam alignment with respect to the guide axis. In general, the precision scales favorably with the pulse area; however, a number of kicks that can be applied are limited by the total interrogation time of interferometer.

In conclusion, we demonstrated an application for de Broglie wave atom interferometer to study reach ADKR phenomena. We showed nearly perfect coherence preservation of matter waves perturbed by a train of optical lattice pulses when the time between successive pulses is a half integer multiple of the Talbot time corresponding to the quantum resonance. The combination of interferometry and ADKR may provide a method for an accurate measurement of recoil frequency. Finally, we note that our scheme can be approached from the multipulse atom interferometer point of view to study an external state de Broglie atom interferometer with more than four pulses [25].

A.T. thanks G. M. Zaslavsky and T. Sleator for stimulating discussions. We acknowledge the financial support from the DARPA under DOD, the ONR, the U.S. Department of the Army (Agreement No. W911NF-07-1-0496), and the Draper Laboratory.
[1] F. L. Moore, J. C. Robinson, C. F. Bharucha, B. Sundaram, and M. G. Raizen, Phys. Rev. Lett. 75, 4598 (1995).

[2] F. M. Izrailev and D. L. Shepelyansky, Dokl. Akad. Nauk SSSR 249, 1103 (1979); Theor. Math. Phys. 43, 553 (1980).

[3] M. K. Oberthaler, R. M. Godun, M. B. d'Arcy, G. S. Summy, and K. Burnett, Phys. Rev. Lett. 83, 4447 (1999); M. B. d'Arcy, R. M. Godun, M. K. Oberthaler, D. Cassettari, and G. S. Summy, ibid. 87, 074102 (2001).

[4] S. Schlunk et al., Phys. Rev. Lett. 90, 054101 (2003).

[5] F. L. Moore, J. C. Robinson, C. Bharucha, P. E. Williams, and M. G. Raizen, Phys. Rev. Lett. 73, 2974 (1994)

[6] C. F. Bharucha et al., Phys. Rev. E 60, 3881 (1999).

[7] D. A. Steck et al., Science 293, 274 (2001).

[8] S. Fishman, I. Guarneri, and L. Rebuzzini, Phys. Rev. Lett. 89, 084101 (2002).

[9] S. Wimberger, I. Guarneri, and S. Fishman, Phys. Rev. Lett. 92, 084102 (2004).

[10] The decoherence due to one pulse was studied by M. S. Chapman et al., Phys. Rev. Lett. 75, 3783 (1995); T. Kohno, S. Suzuki, and K. Shimizu, Phys. Rev. A 76, 053624 (2007); S. Beattie et al., ibid. 79, 021605(R) (2009).

[11] S. Wimberger, M. Sadgrove, S. Parkins, and R. Leonhardt, Phys. Rev. A 71, 053404 (2005).

[12] I. Guarneri and L. Rebuzzini, Phys. Rev. Lett. 100, 234103 (2008); M. Saunders, P. L. Halkyard, S. A. Gardiner, and K. J. Challis, Phys. Rev. A 79, 023423 (2009).

[13] M. Weitz, B. C. Young, and S. Chu, Phys. Rev. Lett. 73, 2563 (1994); S. Gupta, K. Dieckmann, Z. Hadzibabic, and D. E. Pritchard, ibid. 89, 140401 (2002); P. Clade et al., ibid. 96,
033001 (2006)

[14] S. Wu, E. Su, and M. Prentiss, Phys. Rev. Lett. 99, 173201 (2007).

[15] S. B. Cahn et al., Phys. Rev. Lett. 79, 784 (1997).

[16] D. V. Strekalov, A. Turlapov, A. Kumarakrishnan, and T. Sleator, Phys. Rev. A 66, 023601 (2002).

[17] A similar description of QR by M. Lepers, V. Zehnle, and J. C. Garreau, Phys. Rev. A 77, 043628 (2008).

[18] S. Wu, A. Tonyushkin, and M. Prentiss, e-print arXiv:0801.0475.

[19] The effect of amplitude noise on mean energy resonances was experimentally studied by D. A. Steck, V. Milner, W. H. Oskay, and M. G. Raizen, Phys. Rev. E 62, 3461 (2000); M. Sadgrove, A. Hilliard, T. Mullins, S. Parkins, and R. Leonhardt, Phys. Rev. E 70, 036217 (2004); and theoretically by S. Brouard and J. Plata, J. Phys. A 36, 3745 (2003); S. Wimberger et al., Nonlinearity 16, 1381 (2003).

[20] S. Wayper et al., EPL 79, 60006 (2007).

[21] A. Tonyushkin and T. Sleator, Phys. Rev. A 74, 053615 (2006).

[22] C. Ryu et al., Phys. Rev. Lett. 96, 160403 (2006); J. F. Kanem, S. Maneshi, M. Partlow, M. Spanner, and A. M. Steinberg, ibid. 98, 083004 (2007).

[23] If we allow $\theta$ to be random during the sequence, a different scaling law is expected $[9,19]$.

[24] G. Campbell et al., Phys. Rev. Lett. 94, 170403 (2005); S. Beattie et al., Phys. Rev. A 77, 013610 (2008).

[25] A. Tonyushkin and M. Prentiss, Phys. Rev. A 78, 053625 (2008). 